\title{
The In-Gap Charge Current through the Correlated Quantum Dot Hybridized with Superconductor
}

\author{
A. Donabidowicz And T. Domański \\ Institute of Physics, M. Curie-Skłodowska University \\ pl. M. Curie-Skłodowskiej 1, 20-031 Lublin, Poland
}

\begin{abstract}
We explore the Andreev tunnelling through the strongly correlated quantum dot embedded between the normal and superconducting electrodes. For a small external voltage $|e V|<\Delta_{\text {s }}$ the electron arriving from the normal lead can be converted into a pair on the quantum dot and further propagates in the superconducting lead while simultaneously the hole is reflected back to the normal electrode. Conductance of such anomalous current is very sensitive to the particle-hole mixing of the quantum dot spectrum. We analyze the influence of the proximity effect and the Coulomb interactions on the differential Andreev conductance focusing on the extreme limit $\Delta_{\mathrm{s}} \rightarrow \infty$.
\end{abstract}

PACS numbers: 73.23.-b, 74.20.Fg, 74.45.+c

\section{Introduction}

The recent technological progress of nanoscopic devices with ultrasmall islands of atoms where a number of electrons can be varied in a controlled way has attracted a considerable interest. The Coulomb interactions along with the Pauli principle have there a strong influence on the charge and heat currents transmitted through such zero-dimensional quantum dots (QDs). Upon applying an external bias $V$ the differential conductance was found to have an oscillating behavior due to the Coulomb blockade. Moreover, the correlations can lead to formation of the low temperature Kondo resonance showing up by the perfect unitary limit conductance.

In case when the QD is placed at interface with the superconducting electrode there arise some qualitatively new effects related to the collective behavior of electrons bound in the Cooper pairs. We address here such issue. In particular, we show that the order parameter of the superconducting electrode extends onto the QD and at low voltages $|e V| \ll \Delta_{\mathrm{s}}$ this allows for a charge transport via the Andreev reflections. The differential conductance of such current takes either the 
two-peak (for $U=0$ ) or four-peak (when $U \neq 0$ ) structure because the particle and hole excitations are mixed on the QD. Our study here is complementary to some previous works on this subject $[1-5]$.

\section{The single impurity model}

For description of the QD hybridized with one normal $(\beta=\mathrm{N})$ and one superconducting $(\beta=\mathrm{S})$ electrode we exploit the single impurity model

$$
\hat{H}=\hat{H}_{\mathrm{N}}+\hat{H}_{\mathrm{S}}+\sum_{\sigma} \varepsilon_{d} \hat{d}_{\sigma}^{\dagger} \hat{d}_{\sigma}+U \hat{n}_{d \uparrow} \hat{n}_{d \downarrow}+\sum_{\boldsymbol{k}, \sigma, \beta}\left(V_{\boldsymbol{k} \beta} \hat{d}_{\sigma}^{\dagger} \hat{c}_{\boldsymbol{k} \sigma \beta}+\text { h.c. }\right),
$$

where $d_{\sigma}\left(d_{\sigma}^{\dagger}\right)$ are the annihilation (creation) operators of the electron with spin $\sigma=\uparrow$ or $\downarrow$. The energy level is denoted by $\varepsilon_{d}, U>0$ corresponds to the on-dot Coulomb repulsion and the last terms in (1) describe a hybridization of the QD with itinerant electrons of the leads.

We shall treat here the normal lead as a reservoir of free fermions

$$
\hat{H}_{\mathrm{N}}=\sum_{\boldsymbol{k}, \sigma}\left(\varepsilon_{\boldsymbol{k N}}-\mu_{\mathrm{N}}\right) \hat{c}_{\boldsymbol{k} \sigma \mathrm{N}}^{\dagger} \hat{c}_{\boldsymbol{k} \sigma \mathrm{N}}
$$

with their chemical potential $\mu_{\mathrm{N}}$. For simplicity we focus on the wide band limit $\left|V_{\boldsymbol{k} \mathrm{N}}\right| \ll D$ where $\left|\varepsilon_{\boldsymbol{k}}\right| \leq D$. The other superconducting electrode can be described by the BCS Hamiltonian

$$
\hat{H}_{\mathrm{S}}=\sum_{\boldsymbol{k}, \sigma}\left(\varepsilon_{\boldsymbol{k S}}-\mu_{\mathrm{S}}\right) \hat{c}_{\boldsymbol{k} \sigma \mathrm{S}}^{\dagger} \hat{c}_{\boldsymbol{k} \sigma \mathrm{S}}-\sum_{\boldsymbol{k}}\left(\Delta_{\boldsymbol{k}} \hat{c}_{\boldsymbol{k} \uparrow \mathrm{S}}^{\dagger} \hat{c}_{-\boldsymbol{k}_{\downarrow} \dagger \mathrm{S}}^{\dagger}+\text { h.c. }\right)
$$

assuming the isotropic energy gap $\Delta_{k}=\Delta_{\mathrm{s}}$.

Electrons of the superconducting lead are bound in the Cooper pairs and appearance of the off-diagonal order parameter $\left\langle\hat{c}_{-\boldsymbol{k} \downarrow S} \hat{c}_{\boldsymbol{k} \uparrow S}\right\rangle$ is responsible there for a dissipationless motion of the charge carriers. Below we show that the hybridization $V_{\boldsymbol{k} \mathrm{S}}$ transmits such off-diagonal order also onto the QD. To describe this proximity effect we introduce the matrix Green function

$$
\boldsymbol{G}_{d}(\omega)=-\left(\begin{array}{cc}
\hat{T}_{\tau}\left\langle\hat{d}_{\uparrow}(\tau) \hat{d}_{\uparrow}^{\dagger}\right\rangle & \hat{T}_{\tau}\left\langle\hat{d}_{\uparrow}(\tau) \hat{d}_{\downarrow}\right\rangle \\
\hat{T}_{\tau}\left\langle\hat{d}_{\downarrow}^{\dagger}(\tau) \hat{d}_{\uparrow}^{\dagger}\right\rangle & \hat{T}_{\tau}\left\langle\hat{d}_{\downarrow}^{\dagger}(\tau) \hat{d}_{\downarrow}\right\rangle
\end{array}\right)
$$

(where $\hat{T}_{\tau}$ denotes the time ordering operator) and express the Fourier transform of the Green function (4) by the Dyson equation

$$
\boldsymbol{G}_{d}(\omega)^{-1}=\left(\begin{array}{cc}
\omega-\varepsilon_{d} & 0 \\
0 & \omega+\varepsilon_{d}
\end{array}\right)-\boldsymbol{\Sigma}_{d}^{0}(\omega)-\boldsymbol{\Sigma}_{d}^{U}(\omega),
$$

where $\Sigma_{d}^{0}(\omega)$ accounts for the hybridization of the QD to both electrodes and $\Sigma_{d}^{U}(\omega)$ comes from the Coulomb interactions $U$. The self-energy has been so far estimated by a number of authors using various methods (see e.g. citations in Ref. [4]).

To start our analysis we first recall that the imaginary part of $\boldsymbol{\Sigma}_{d}^{0}(\omega)$ is given by $[6]$ : 


$$
\operatorname{Im}\left\{\Sigma_{d}^{0}(\omega)\right\}=\left(\begin{array}{cc}
-\Gamma_{\mathrm{N}} & \Gamma_{\mathrm{S}} \Delta_{\mathrm{s}} \frac{\Theta\left(|\omega|-\Delta_{\mathrm{s}}\right)}{\sqrt{\omega^{2}-\Delta_{\mathrm{s}}^{2}}} \\
-\Gamma_{\mathrm{S}} \Delta_{\mathrm{s}} \frac{\Theta\left(|\omega|-\Delta_{\mathrm{s}}\right)}{\sqrt{\omega^{2}-\Delta_{\mathrm{s}}^{2}}} & -\Gamma_{\mathrm{N}}
\end{array}\right),
$$

where $\Gamma_{\beta}$ stands for the hybridization couplings defined by

$$
2 \pi \sum_{\boldsymbol{k}}\left|V_{\boldsymbol{k} \beta}\right|^{2} \delta\left(\omega-\varepsilon_{\boldsymbol{k} \beta}\right) \equiv \begin{cases}\Gamma_{\beta} & \text { for }-D<\omega<D \\ 0 & \text { elsewhere. }\end{cases}
$$

One can determine the real part using the Kramers-Krönig relation and we have illustrated its $\omega$-dependence in Fig. 3 of our paper [6]. For a simple understanding of the relevant physics we now follow Tanaka et al. [5] and focus on the extreme limit of the large energy gap $\Delta_{\mathrm{s}}$. For energies deep inside the superconducting gap $\Delta_{\mathrm{S}} \gg|\omega|$ we obtain

$$
\boldsymbol{\Sigma}_{d}^{0}(\omega)=\left(\begin{array}{cc}
-\mathrm{i} \Gamma_{\mathrm{N}} & -\Gamma_{\mathrm{S}} \frac{2}{\pi} \operatorname{atan}\left(\frac{D}{\Delta_{\mathrm{s}}}\right) \\
-\Gamma_{\mathrm{S}} \frac{2}{\pi} \operatorname{atan}\left(\frac{D}{\Delta_{\mathrm{s}}}\right) & -\mathrm{i} \Gamma_{\mathrm{N}}
\end{array}\right),
$$

where the off-diagonal terms simplify to $-\Gamma_{\mathrm{s}}[5]$ when $\Delta_{\mathrm{s}} \ll D$. Substituting this result to the Dyson equation (5) we can analytically determine the matrix Green function of the noninteracting QD. We obtain the BCS-structure

$$
\begin{aligned}
& \boldsymbol{G}_{d, 11}(\omega)=\frac{\frac{1}{2}\left(1+\varepsilon_{d} / E\right)}{\omega+\mathrm{i} \Gamma_{\mathrm{N}}-E}+\frac{\frac{1}{2}\left(1-\varepsilon_{d} / E\right)}{\omega+\mathrm{i} \Gamma_{\mathrm{N}}+E}, \\
& \boldsymbol{G}_{d, 12}(\omega)=\frac{-\Gamma_{\mathrm{s}} / 2 E}{\omega+\mathrm{i} \Gamma_{\mathrm{N}}-E}+\frac{\Gamma_{\mathrm{s}} / 2 E}{\omega+\mathrm{i} \Gamma_{\mathrm{N}}+E}
\end{aligned}
$$

with $E=\sqrt{\varepsilon_{d}^{2}+\Gamma_{\mathrm{s}}^{2}}$. The spectral function $\rho_{d}(\omega)=-\frac{1}{\pi} \operatorname{Im} \boldsymbol{G}_{d, 11}(\omega)$ is thus characterized by two peaks because the particle and hole excitations on the QD become mixed with one another [6]. In Fig. 1 we show $\rho_{d}(\omega)$ for the energy level located in the center of the superconductor gap $\varepsilon_{d}=\mu_{\beta}$. Distance between the peaks depends on $\Gamma_{\mathrm{S}}$, the broadenig on $\Gamma_{\mathrm{N}}[5]$ and the weights of the peaks change upon varying $\varepsilon_{d}$ as shown in Fig. 4 in Ref. [6].

The BCS structure of the Green function (10) leads to apppearance of the off-diagonal order parameter $\left\langle\hat{d}_{\downarrow} \hat{d}_{\uparrow}\right\rangle$ (see the left part in Fig. 2). This means that the proximity effects convert the QD into a superconducting grain. For the equilibrium situation we estimate

$$
\left\langle\hat{d}_{\uparrow} \hat{d}_{\downarrow}\right\rangle=\frac{\Gamma_{\mathrm{s}}}{2 E}[F(E)-F(-E)]
$$

where $F(E)=\int_{-D}^{D} \frac{\mathrm{d} \omega}{\pi} \frac{\Gamma_{\mathrm{N}}}{(\omega-E)^{2}+\Gamma_{\mathrm{N}}^{2}}\left(1+\mathrm{e}^{E / k_{\mathrm{B}} T}\right)^{-1}$. In Fig. 2 we show that optimal conditions for the off-diagonal order parameter (11) as well as for a magnitude of the zero bias Andreev conductance correspond to $\varepsilon_{d}$ located near the center of the energy gap.

\section{The generalized Schrieffer-Wolf transformation}

To have an independent evidence signifying the appearance of the order parameter on the QD we can construct the canonical transformation 


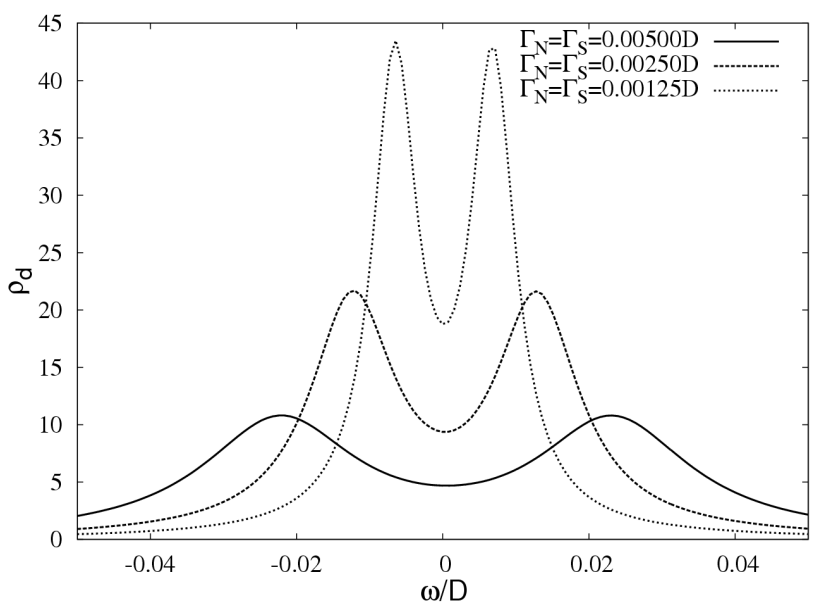

Fig. 1. Splitting of the QD spectral function because of the proximity effect determined in the equilibrium situation $\varepsilon_{d}=\mu_{\mathrm{N}}=\mu_{\mathrm{S}}$ for several values of the hybridization coupling $\Gamma_{\beta}=2 \pi \sum_{\boldsymbol{k}}\left|V_{\boldsymbol{k} \beta}\right|^{2} \delta\left(\omega-\varepsilon_{\boldsymbol{k} \beta}\right)$. We used for calculations $\Delta=0.1 D, U=0$ and set $D$ as a unit for energies.
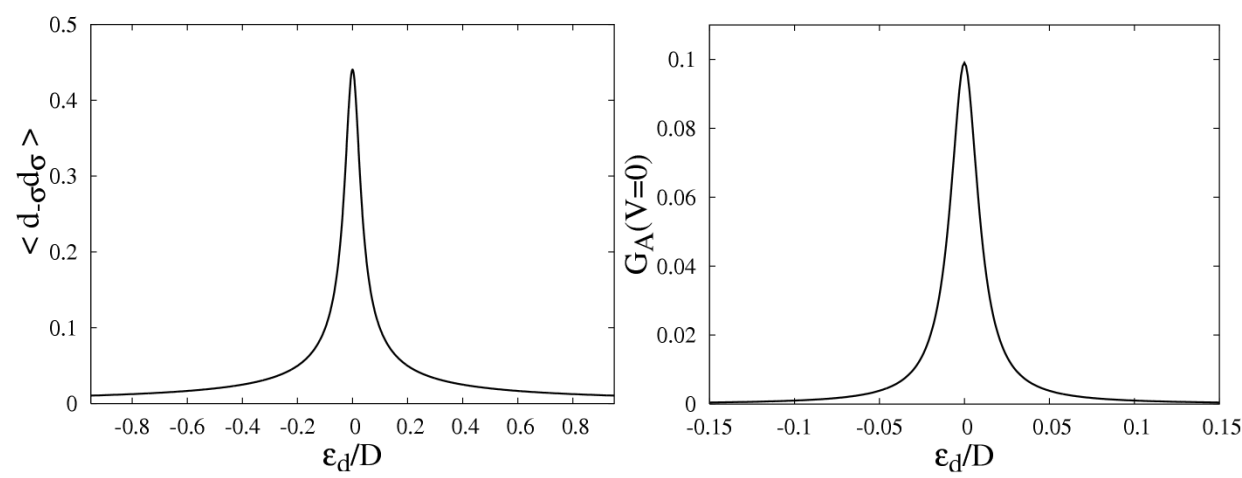

Fig. 2. Left part: The off-diagonal order parameter $\left\langle\hat{d}_{\downarrow} \hat{d}_{\uparrow}\right\rangle$ of the uncorrelated QD with $\Gamma_{\mathrm{N}}=0.001 D, \Gamma_{\mathrm{S}}=0.01$ in the limit $\Delta_{\mathrm{s}} \rightarrow \infty$. To carry out the statistical average we assumed the equilibrium situation $V=0$. Right part: The zero bias conductance of the in-gap Andreev current (20) in units of $4 e^{2} / h$.

$$
\hat{H} \longrightarrow \hat{\tilde{H}}=\mathrm{e}^{\hat{S}} \hat{H} \mathrm{e}^{-\hat{S}}
$$

eliminating the hybridization $V_{\boldsymbol{k}, \mathrm{S}}$ up to quadratic terms. For this purpose we extend the Schrieffer and Wolf treatment [7] which has been originally designed for the impurity embedded in a conduction band. We choose the following anti-Hermitian operator $\hat{S}=\left(\hat{S}^{0}+\hat{S}^{U}\right)-\left(\hat{S}^{0}+\hat{S}^{U}\right)^{\dagger}$, where

$$
\hat{S}^{0}=\sum_{\boldsymbol{k}} V_{\boldsymbol{k} \mathrm{S}}\left[\frac{\xi_{\boldsymbol{k}}+\varepsilon_{d}}{E_{\boldsymbol{k}}^{2}-\varepsilon_{d}^{2}}\left(\hat{c}_{\boldsymbol{k} \uparrow}^{\dagger} \hat{d}_{\uparrow}+\hat{c}_{\boldsymbol{k} \downarrow}^{\dagger} \hat{d}_{\downarrow}\right)+\frac{\Delta_{\mathrm{S}}^{*}}{E_{\boldsymbol{k}}^{2}-\varepsilon_{d}^{2}}\left(\hat{c}_{\boldsymbol{k} \uparrow} \hat{d}_{\downarrow}-\hat{c}_{-\boldsymbol{k} \downarrow} \hat{d}_{\uparrow}\right)\right],
$$


where $E_{\boldsymbol{k}}=\sqrt{\xi_{\boldsymbol{k}}^{2}+\left|\Delta_{\mathrm{s}}\right|^{2}}$. The other term $\hat{S}^{U}$ (whose explicit form will be discussed elsewhere) comes from the Coulomb correlations $U$ and is crucial for description of the effective exchange interactions leading to the Kondo effect [7]. Because of a lack of space we postpone such aspects for the separate publication. Here we briefly focus on studying the terms which are distinct from the usual Schrieffer-Wolf result [7]. In the transformed Hamiltonian $\hat{\tilde{H}}$ we obtain the following change of the QD part:

$$
\hat{H}_{d} \rightarrow \hat{\tilde{H}}_{d}=\sum_{\sigma} \tilde{\varepsilon}_{d} \hat{d}_{\sigma}^{\dagger} \hat{d}_{\sigma}+U \hat{n}_{d \uparrow} \hat{n}_{d \downarrow}-\left(\Delta_{d}^{*} \hat{d}_{\downarrow} \hat{d}_{\uparrow}+\Delta_{d} \hat{d}_{\uparrow}^{\dagger} \hat{d}_{\downarrow}^{\dagger}\right),
$$

where

$$
\Delta_{d}=\sum_{k, \sigma}\left|V_{k \mathrm{~S}}\right|^{2} \frac{\Delta_{\mathrm{s}}}{E_{\boldsymbol{k}}^{2}-\varepsilon_{d}^{2}} .
$$

We notice that indeed the QD can be treated as a superconducting grain with an effective gap parameter $\Delta_{\mathrm{d}}$. For the case of large $\Delta_{\mathrm{s}}$ we estimate

$$
\Delta_{d} \simeq-\Gamma_{\mathrm{S}} \frac{1}{\pi} \int_{-D}^{D} \frac{\Delta_{\mathrm{S}}}{\varepsilon^{2}+\Delta_{\mathrm{S}}^{2}}=-\Gamma_{\mathrm{S}} \frac{2}{\pi} \operatorname{atan}\left(\frac{D}{\Delta_{\mathrm{s}}}\right),
$$

which is identical with the off-diagonal terms of the self-energy (8). Furthermore, for $D \gg \Delta_{\mathrm{S}}$ our result (16) reduces to $-\Gamma_{\mathrm{S}}$ as has been independently pointed out by Tanaka et al. [5].

\section{Influence of the Coulomb interactions}

In a presence of the Coulomb interactions we must take into account the matrix self-energy $\boldsymbol{\Sigma}_{d}^{U}(\omega)$. It has been proved by Affleck et al. [10] and by Oguri et al. [11] that in the limit $\Delta_{\mathrm{s}} \rightarrow \infty$ the effect of $U$ affects only the diagonal parts of the self-energy. In other words, the Coulomb interactions do not modify the on-dot energy gap $\Delta_{d}$. Following such argumentation we propose here to use a simple substitution for $\boldsymbol{\Sigma}_{d}^{U}(\omega)$ based on the atomic solution

$$
\Sigma_{11}^{U}(\omega)=\frac{U\left\langle n_{d \sigma}\right\rangle\left(\omega-\varepsilon_{d}\right)}{\omega-\varepsilon_{d}-U+U\left\langle n_{d \sigma}\right\rangle}=-\Sigma_{22}^{U}(-\omega)
$$

and $\boldsymbol{\Sigma}_{12}^{U}(\omega)=0$. This yields the following Green function:

$$
\begin{aligned}
\boldsymbol{G}_{d, 11}(\omega) & =\frac{\boldsymbol{G}_{\mathrm{at}, 22}(\omega)^{-1}}{\boldsymbol{G}_{\mathrm{at}, 11}(\omega)^{-1} \boldsymbol{G}_{\mathrm{at}, 22}(\omega)^{-1}-\Gamma_{\mathrm{S}}^{2}}, \\
\boldsymbol{G}_{d, 12}(\omega) & =\frac{\Gamma_{\mathrm{S}}}{\boldsymbol{G}_{\mathrm{at}, 11}(\omega)^{-1} \boldsymbol{G}_{\mathrm{at}, 22}(\omega)^{-1}-\Gamma_{\mathrm{S}}^{2}},
\end{aligned}
$$

where $\boldsymbol{G}_{\mathrm{at}, 11}(\omega)=\frac{1-\left\langle n_{d \downarrow}\right\rangle}{\omega+i \Gamma_{\mathrm{N}}-\varepsilon_{d}}+\frac{\left\langle n_{d \downarrow}\right\rangle}{\omega+\mathrm{i} \Gamma_{\mathrm{N}}-\varepsilon_{d}-U}$ and $\boldsymbol{G}_{\mathrm{at}, 22}(\omega)=-\boldsymbol{G}_{\mathrm{at}, 11}(-\omega)$. This result shows that the Coulomb interactions have a strong effect on the spectral function. The two peak spectrum of the uncorrelated QD (see Fig. 1) is replaced by the four-peak structure. This kind of result has been previously obtained by Sun et al. [8] using a different method. In a forthcoming paper we shall discuss 
in some more detail the issue of correlations, in particular explaining also their influence on the Kondo resonance.

\section{The in-gap charge current}

To compute the charge current $J(V)=-e \frac{\mathrm{d}}{\mathrm{d} t} \sum_{\boldsymbol{k}, \sigma}\left\langle\hat{c}_{\boldsymbol{k} \mathrm{N} \sigma}^{\dagger} \hat{c}_{\boldsymbol{k N} \sigma}\right\rangle$ one needs to use the nonequilibrium Keldysh-Green functions. Following the standard procedure [9] we determine $J(V)$ focusing on the limiting case when $|e V| \ll \Delta_{\mathrm{s}}$. The in-gap current can be transmitted only through the Andreev reflections. Physically this happens when the incident electron from the normal lead converts into a pair on the QD and further propagates to the superconducting lead with a simultaneous reflection of the hole back to the normal electrode. The Andreev current is formally given by [2]:

$$
J_{\mathrm{A}}(V)=\frac{2 e \Gamma_{\mathrm{N}}^{2}}{h} \int \mathrm{d} \omega T_{\mathrm{A}}(\omega)[f(\omega-e V)-f(\omega+e V)],
$$

where $f(x)=\left[1+\exp \left(x / k_{\mathrm{B}} T\right)\right]^{-1}$. The corresponding transmittance depends on the off-diagonal Green function

$$
T_{\mathrm{A}}(\omega)=\left|G_{d, 12}(\omega)\right|^{2} .
$$

Thus calculated Andreev conductance for the uncorrelatedquantum dot is illustrated in Fig. 3.

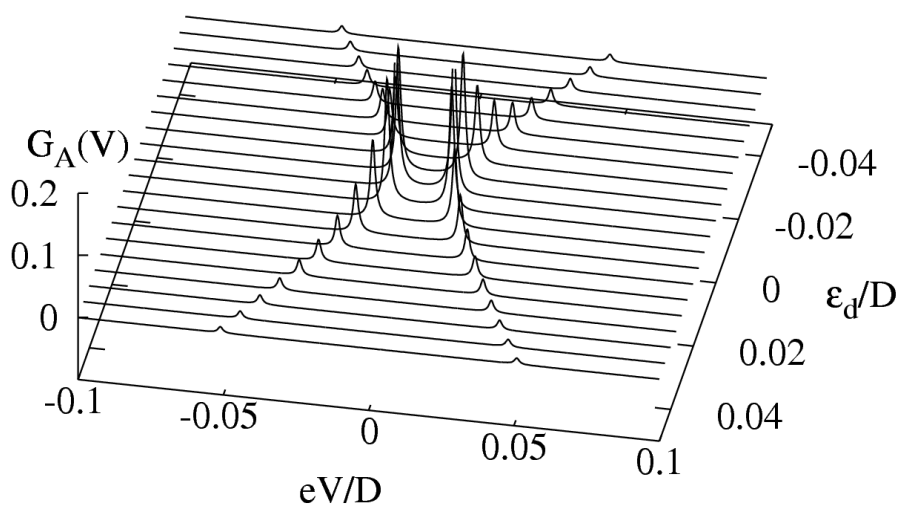

Fig. 3. Differential conductance of the Andreev current (20) expressed in units of $4 e^{2} / h$. We have done the calculations using $U=0, \Gamma_{\mathrm{N}}=0.001 D$ and $\Gamma_{\mathrm{S}}=0.01 D$.

In Fig. 4 we show the differential conductance $G_{\mathrm{A}}(V)=\mathrm{d} J_{\mathrm{A}}(V) / \mathrm{d} V$ computed for the correlated QD whose single energy $\varepsilon_{d}=-U / 2$. For this symmetric case $\left\langle n_{d \sigma}\right\rangle=0.5$ we obtain that the Andreev conductance has a four-peak structure, similarly as the corresponding QD spectrum [8]. These peaks result from a combined effect of the particle-hole mixing along with the Coulomb interactions. 


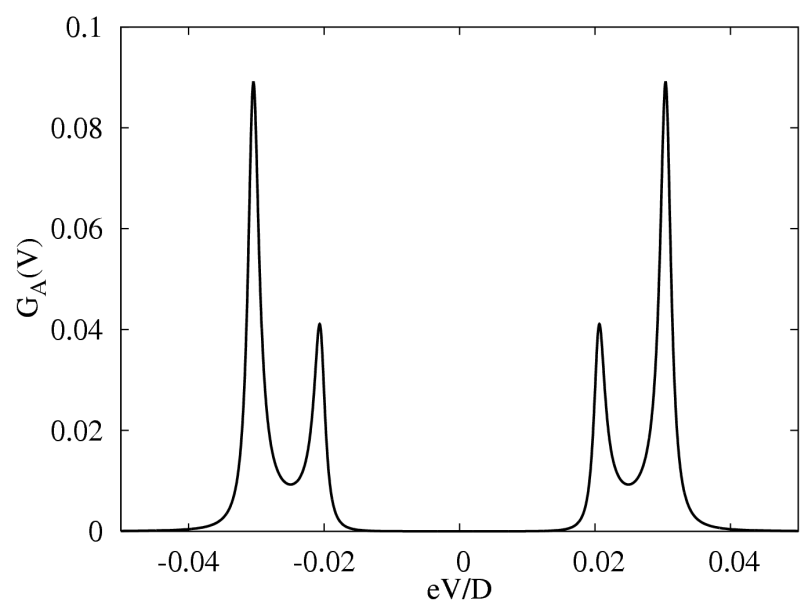

Fig. 4. Voltage dependence of the in-gap Andreev conductance $G_{\mathrm{A}}(V)$ for the correlated quantum $\operatorname{dot} U=0.05 D$ with the single energy level $\varepsilon_{\mathrm{d}}=-U / 2$ and the coupling constants $\Gamma_{\mathrm{S}}=0.01 D, \Gamma_{\mathrm{N}}=0.001 D$. The conductance is expressed in units of $2 e^{2} / h$.

\section{Conclusion}

We have studied the in-gap charge transport through the quantum dot hybridized with one normal and one superconducting lead. At low voltages the current flows through the anomalous Andreev channel owing to the proximity effect. We have shown that the uncorrelated $\mathrm{QD}(U=0)$ is characterized by the two-peak differential conductance due to the mixed particle hole excitations of the QD. The distance between such peaks depends solely on the hybridization $\Gamma_{\mathrm{S}}$, a specific value of the energy gap $\Delta_{\mathrm{S}}$ is irrelevant there. In presence of the Coulomb interactions the Andreev conductance splits into the four-peak structure. Correlations are expected to have an additional influence on $G_{\mathrm{A}}(V)$ upon entering the Kondo regime [6] but this subtle issue is beyond the scope of the present work.

\section{Acknowledgments}

We kindly acknowledge the discusions with Prof. K.I. Wysokiński and Dr. M. Krawiec. This work is partly supported by the Polish Ministry of Science and Education under the grants NN202187833 and NN202373333.

\section{References}

[1] R. Fazio, R. Raimondi, Phys. Rev. Lett. 80, 2913 (1998); Phys. Rev. Lett. 82, 4950 (1998); P. Schwab, R. Raimondi, Phys. Rev. B 59, 1637 (1999).

[2] Q.-F. Sun, J. Wang, T.-H. Lin, Phys. Rev. B 59, 3831 (1999).

[3] A.A. Clerk, A. Ambegaokar, S. Herschfield, Phys. Rev. B 61, 3555 (2000).

[4] M. Krawiec, K.I. Wysokiński, Supercond. Sci. Technol. 17, 103 (2004).

[5] Y. Tanaka, N. Kawakami, A. Oguri, J. Phys. Soc. Jpn. 76, 074701 (2007). 
[6] T. Domański, A. Donabidowicz, K.I. Wysokiński, Phys. Rev. B 76, 104514 (2007).

[7] J.R. Schrieffer, P.A. Wolf, Phys. Rev. 149, 491 (1966).

[8] Q.-F. Sun, H. Guo, T.-H. Lin, Phys. Rev. Lett. 87, 176601 (2001).

[9] Y. Meir, N.S. Wingreen, Phys. Rev. Lett. 67, 2512 (1992); Y. Meir, N.S. Wingreen, P.A. Lee, Phys. Rev. Lett. 70, 2601 (1993).

[10] I. Affleck, J.-S. Caux, A.M. Zagoskin, Phys. Rev. B 62, 1433 (2000).

[11] A. Oguri, Y. Tanaka, A.C. Hewson, J. Phys. Soc. Jpn. 73, 2494 (2004). 